\title{
The role of the glycosyl moiety of myricetin derivatives in anti-HIV-1 activity in vitro
}

Joseph T. Ortega ${ }^{1}$, Alirica I. Suárez², Maria L. Serrano ${ }^{3}$, Jani Baptista ${ }^{2}$, Flor H. Pujol ${ }^{1}$ and Hector R. Rangel ${ }^{\text {* }^{*} \text { (D) }}$

\begin{abstract}
Background: Plant extracts are sources of valuable compounds with biological activity, especially for the antiproliferative activity against pathogens or tumor cells. Myricetin is a flavonoid found in several plants that has been described as an inhibitor of Human immunodeficiency virus type 1 (HIV-1) through its action against the HIV reverse transcriptase, but myricetin derivatives have not been fully studied. The aim of this study was to evaluate the antiHIV-1 activity of glycosylated metabolites obtained from Marcetia taxifolia and derived from myricetin: myricetin rhamnoside and myricetin 3-(6-rhamnosylgalactoside).
\end{abstract}

Methods: Compounds were obtained from organic extracts by maceration of aerial parts of M. taxifolia. All biological assays were performed in the MT4 cell line. Antiviral activity was measured as inhibition of p24 and reverse transcriptase with a fluorescent assay.

Results: Both flavonoids have antiviral activity in vitro, with an EC50 of $120 \mu \mathrm{M}$ for myricetin 3-rhamnoside (MR) and $45 \mu \mathrm{M}$ for myricetin 3-(6-rhamnosylgalactoside) (MRG), both significantly lower than the EC50 of myricetin (230 $\mu \mathrm{M})$. Although both compounds inhibited the reverse transcriptase activity, with an IC50 of $10.6 \mu \mathrm{M}$ for MR and $13.8 \mu \mathrm{M}$ for MRG, myricetin was the most potent, with an IC50 of $7.6 \mu \mathrm{M}$, and an inhibition greater than $80 \%$. Molecular docking approach showed correlation between the free energy of binding with the assays of enzyme inhibition.

Conclusions: The results suggest that glycosylated moiety might enhance the anti-HIV-1 activity of myricetin, probably by favoring the internalization of the flavonoid into the cell. The inhibition of the HIV-1 reverse transcriptase is likely responsible for the antiviral activity.

Keywords: HIV-1, Reverse transcriptase, Flavonoids, Myricetin, Glycosyl flavonoids, HIV RT docking

\section{Background}

The AIDS epidemic is the result of one of the most important viral infections affecting humans. As there is no vaccine currently available, the antiviral drugs constitute the only way to slow the progression of the disease $[1,2]$. The appearance of drug resistance, serious side effects of existing drugs have promoted the search for new anti-HIV agents [3].

\footnotetext{
*Correspondence: hrangel2006@gmail.com

${ }^{1}$ Laboratorio de Virología Molecular, Centro de Microbiología y Biología

Celular, Instituto Venezolano de Investigaciones Científicas, Caracas,

Venezuela

Full list of author information is available at the end of the article
}

Plants are a rich source of new bioactive compounds [4]. Flavonoids, a large group of polyphenolic compound, are known for their bioactive properties and are widely distributed in the vegetal kingdom [5]. The range of their biological properties include anti-allergic, antibacterial, antidiabetic, anti-inflammatory and antiviral activities [6, 7].

The Melastomataceae are plants common in the tropical and mountainous areas. This family is abundantly distributed in South America, Southeast Asia and southern China [8]. Marcetia is a neotropical genus with 44 described species, distributed from Venezuela to Uruguay, with most of the species found in Southern Brazil and Uruguay [9]. The extract of Marcetia taxifolia has 
been shown to have antimicrobial activities, and the compounds responsible for this effect are flavonoids, particularly myricetin [10]. Pasetto et al. [11] and Ono et al. [12] determined that myricetin and quercetin inhibited HIV-1 proliferation and the HIV-1 reverse transcriptase (RT). The RT plays an essential role in the HIV life cycle and is one of the principal targets for several anti HIV-1 drugs in clinical use [13]. Some flavonoids have been characterized as nonnucleoside inhibitors (NNRTIs) that bind to a site in the p66 subunit of the HIV-1 RT p66/p51 heterodimer, situated approximately $10 \AA$ from the RT active site $[14,15]$. Ortega et al. [16], found that quercetin, a glycosylated derivative of myricetin, exhibited an improved antiviral activity and suggested that the enhanced activity was due to the glycosyl moiety.

The aim of this study was to evaluate the anti-HIV-1 activity in vitro of glycosylated metabolites of myricetin obtained from Marcetia taxifolia extracts: myricetin 3-rhamnoside (MR) and myricetin 3-(6-rhamnosylgalactoside) (MRG). These compounds contain one and two glycosides respectively, as substituents on the myricetin backbone (Fig. 1). In order to determine the possible role of the glycosyl moiety on antiviral activity we used a molecular docking approach to compare the binding of the glycosylated compounds and the aglycone myricetin to HIV-1 RT.

\section{Methods}

\section{Plant material and extraction}

The plant Marcetia taxifolia (A.St.-Hil.) DC., was collected in the Amazonas State of Venezuela, and its botanical identity was confirmed and authenticated by Dr. Stephen Tillett. A voucher of the collection was archived with the code MYF 28418 in the "Herbario Víctor Manuel Ovalles" of the School of Pharmacy, Universidad Central de Venezuela. Extraction and separation of compounds obtained from the aerial parts of $M$. taxifolia was performed as described previously [17]. Two glycosylated compounds derived from myricetin: myricetin 3-rhamnoside and myricetin 3-(6-rhamnosylgalactoside) were evaluated, along with the commercially available aglycone myricetin (Sigma-Aldrich, USA).

\section{Cells and virus}

HIV-1 (HTLV-IIIB/H9) and MT4 cells were obtained from the NIH AIDS Research and Reference Reagent Program. The cells were grown in RPMI-1640 medium supplemented with 10\% FBS and penicillin/streptomycin and splitted every 3 days.

\section{Cytotoxicity assay}

MT4 cells were seeded in 96 wells/plate at a density of 30,000 cells/well, and different concentrations of the compounds were added. After $24 \mathrm{~h}$ the cultures were evaluated with MTT cell proliferation assay (SigmaAldrich, USA) to determine the percent of live cells under the different conditions evaluated.

\section{Antiviral activity}

Different concentrations of the compounds and HIV1 , at an MOI of 0.03 , were added simultaneously to the wells of a 96 wells plate containing 30,000 cells/well. The virus production was evaluated at $72 \mathrm{~h}$ post infection by detecting the viral antigen p24 with an in-house ELISA [18]. The results were expressed relative to the control of untreated HIV-1 infected cells. Selectivity Index value (SI) was determined as the ratio of CC50 to EC50 for each compound.

\section{RT inhibition assay}

The inhibitory activity of compounds against HIV-1 RT was evaluated by using the EnzChek Reverse Transcriptase Assay kit (Molecular Probes, Inc., USA). Purified RT enzyme purchased from Worthington Biochemical Corporation and Nevirapine were used as controls for enzyme and antiviral drug respectively. Statistical analysis (ANOVA) of the results from at least three independent experiments was performed using
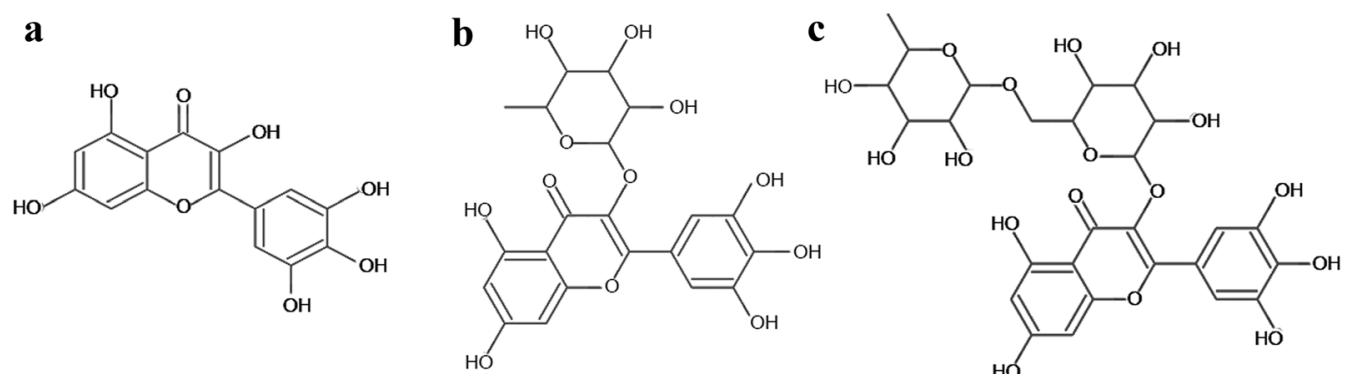

Fig. 1 Chemical structure of the myricetin and derivatives a Myricetin (M), b myricetin 3-rhamnoside (MR) and c myricetin 3-(6-rhamnosylgalactoside) (MRG) 
Prism GraphPad 6 version 6.01 ( $\mathrm{p} \leq 0.05$ was used as the level of significance).

\section{Molecular docking}

The coordinates of wild-type HIV-1 reverse transcriptase (RT) bound with DNA and the NNRTI Nevirapine at $2.85 \AA$ resolution was obtained from the protein data bank [19], PDB code 3V81 [20]. The inhibitor, the DNA molecule and all crystallographic water molecules were removed from the coordinate set: hydrogen atoms were added and partial charges were assigned to all atoms. The enzyme was then submitted to restrained molecular mechanics refinement with NAMD [21], using the CHARMM22 force field. The binding site was delimited according to previous studies reporting key residues of the enzyme and the 3D structure of each inhibitor was obtained from the PubChem database [22]. The molecular docking was performed with AutoDock 4/VegaZZ 3.1.0.21 and 30 runs were conducted for each compound. The results were prioritized according to the predicted free energy of binding in $\mathrm{kcal} / \mathrm{mol}$.

\section{Results}

\section{Cytotoxicity and antiviral activity}

The anti-HIV-1 activity in vitro was evaluated with a wide range of concentrations of the myricetin glycosylated compounds. The EC50 for myricetin 3-rhamnoside was $120 \mu \mathrm{M}$, for myricetin 3-(6-rhamnosylgalactoside) 45 and $230 \mu \mathrm{M}$ for myricetin (Fig. 2a). Neither myricetin nor either of the myricetin derivatives used, exhibited significant cytotoxic activity at the concentrations tested (Fig. 2b). Because the CC50 for all the compounds assayed was out of the range of the evaluated concentrations, (maximum $>300 \mu \mathrm{M}$ ) an exact estimation of the selectivity index (SI) was impossible, and instead a crude estimation was performed by assigning the CC50 as $>300 \mu \mathrm{M}$ and then calculating the EC50 for each compound. The estimated CC50 values thereby obtained were: $\mathrm{IS}_{\mathrm{M}}>1.3$ IS $_{\mathrm{MR}}>2.6$ and $\mathrm{IS}_{\mathrm{MRG}}>7$.

\section{RT inhibition assay}

The capacity of the compounds to inhibit HIV-1 RT was also evaluated. Figure 3 shows that at a concentration higher than $7.5 \mu \mathrm{M}$, myricetin 3-rhamnoside was more potent than myricetin 3-(6-rhamnosylgalactoside), reaching an inhibitory effect over $65 \%$. However, the aglycone myricetin exhibited more RT inhibition at all concentrations evaluated. The IC50 obtained for myricetin was $7.6 \mu \mathrm{M}$, while for myricetin 3-rhamnoside it was $10.6 \mu \mathrm{M}$, and for myricetin 3-(6-rhamnosylgalactoside) $13.8 \mu \mathrm{M}$.

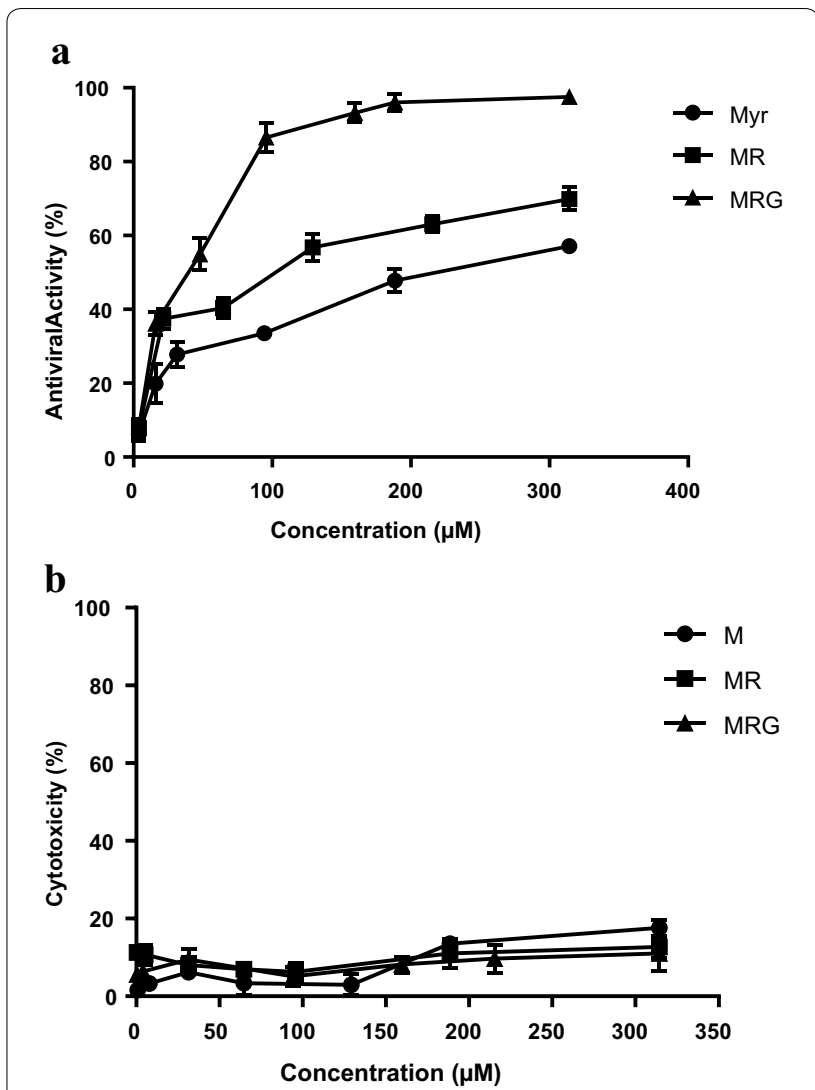

Fig. 2 Antiviral $\mathbf{a}$ and cytotoxic $\mathbf{b}$ activity of compounds evaluated. The percent of cytotoxicity of different concentrations of MR (Square), MRG (Triangle) and M (Circle) are shown as media \pm SEM. Antiviral activity (solid lines) is expressed as the percent inhibition of p24 production relative to control. Results are media $\pm \operatorname{SEM}(n=3)$

\section{Molecular docking}

The potential interactions of the active compounds with the RT enzyme were evaluated by molecular docking. Myricetin exhibited the lowest free energy of binding ( $-6.95 \mathrm{kcal} / \mathrm{mol})$, compared to glycosylated compounds (Table 1). In Fig. 4, which shows the interactions between myricetin and RT residues, there are five hydrogen bonds with residues Lys101 (A), Glu138 (B) and Ile180 (A) and electrostatic interactions $\mathrm{Pi}-\mathrm{Pi}, \mathrm{Pi}-\mathrm{Alkyl}$ and $\mathrm{Pi}-$ sigma with residues Tyr188 (A), Leu100 (A), Val106 (A), Leu234 (A) and Val179 (A). Of the two myricetin glycosides, myricetin 3-rhamnoside exhibited the best free energy of binding to RT, $(-5.02 \mathrm{kcal} / \mathrm{mol})$ and Fig. 4b shows the interactions of myricetin 3-rhamnoside with various amino acid residues in the binding pocket of RT NNRTI inhibitors. Four hydrogen bonds with residues Tyr318 (A), Lys101 (A) and Ile108 (A) were observed, along with electrostatic interactions such as $\mathrm{Pi}-\mathrm{Pi}, \mathrm{Pi}-\mathrm{Alkyl}$, Pi-sigma and Pi-anion with Tyr181 (A), Val 179 (A), Leu100 (A), Glu 138 (B), and Alkyl-Alkyl interactions 


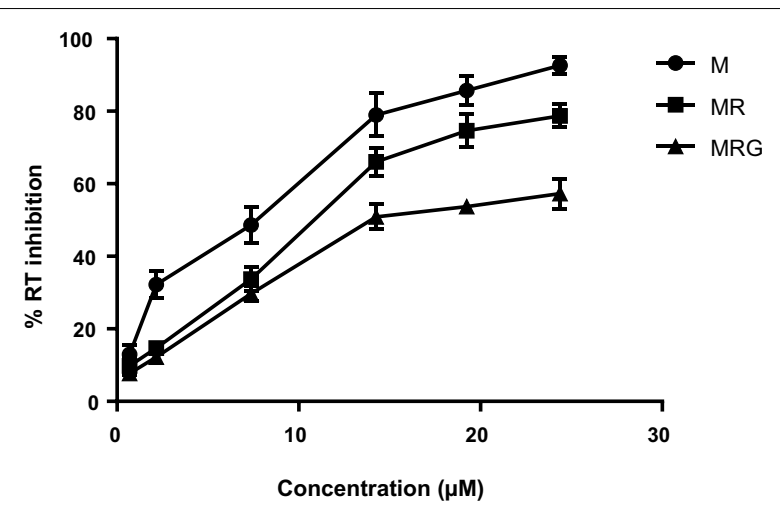

Fig. 3 Reverse transcriptase inhibition. The inhibitory effect of MR (Square) MRG (Triangle) and M (Circle) on recombinant Reverse Transcriptase enzyme $(0.25 \mathrm{Ul} / \mathrm{ml})$, is expressed as the activity of the treated enzyme relative to control w/o treatment. All results were normalized to respective control (reaction buffer)

with the residues Lys103 (A), Val106 (A), Val179 (A) and Pro236 (A). Myricetin 3-(6-rhamnosylgalactoside) showed a higher free energy of binding to RT $(2.38 \mathrm{kcal} /$ $\mathrm{mol}$ ) and fewer interactions than seen with myricetin 3-rhamnoside, two hydrogen bonds with residues Lys101 (A) and Glu138 (A) and several electrostatic interactions $\mathrm{Pi}$-cation and, Pi-Alkyl that occurred with the residues Leu100, Pro321, and Lys101.

\section{Discussion}

Because of several reports describing the activity of other flavonoids against HIV-1 [23-25] we evaluated the activity of glycosylate flavonoids obtained from $M$. taxifolia. The results showed that the antiviral potency of myricetin derived compounds was related to the number of glycosyl residues on the molecule supporting the notion that the addition of glycosides to myricetin could modulate the anti-HIV-1 activity of these compounds.

Flavonoids are generally found in plants and fruits as glycosides and less commonly as aglycones [26]. The glycosilated flavonoids obtained from the diet are hydrolyzed in the intestine and then transported into the cells and systemic circulation. However, it has been described that glycosylated flavonoids may be a substrate of sodium-dependent glucose transporters (SGLT1) in the cell membrane of enterocytes $[27,28]$ and then, once inside the cell, the glycosides are cleaved by a cytosolic beta-glycosidase [29]. This process could contribute to their accumulation within the cell. Consistent with this possibility, it has been shown that two glycosyl derivatives of quercetin (guajaverin and avicularin) have higher antiviral activity than quercetin [16]. Similar results were obtained in this study, with a reduction of two to fivefold of the EC50 with the addition of glycosyl moieties to myricetin.

In contrast to the results observed with the inhibition of HIV-1 replication, RT inhibition was inversely proportional to the glycosylation grade of myricetin [myricetin $(\mathrm{R}=$ aglycone $)>$ myricetin 3-rhamnoside $(\mathrm{R}=$ monoglycosylated) > myricetin 3-(6-rhamnosylgalactoside) $(\mathrm{R}=$ Bi-glycosylated $)]$. These results are in concordance with the free energy of binding obtained in silico, where myricetin exhibited the lowest free energy and MRG the highest. The analysis of the resulting complexes clearly reveals that the binding interactions of these compounds occurred with the residues of the hydrophobic NNRTIbinding pocket, described by Sarafianos et al. [30] as containing residues Leu100, Lys101, Lys103, Val106, Tre107, Val108, Val179, Tyr181, Tyr188, Val189, Gly190, Phe227,

Table 1 Docking analysis of the evaluated compounds

\begin{tabular}{|c|c|c|c|c|}
\hline Mol ID & $\begin{array}{l}\mathrm{EC}_{50} \\
\text { (Elisa } \\
\text { p24) }\end{array}$ & $E B^{a}$ & RT interactions & $\begin{array}{l}\text { Residues ( } \mathrm{H} \text {-bonds) } \\
\text { Number of } \mathrm{H} \text { bonds }\end{array}$ \\
\hline \multirow[t]{2}{*}{ M } & 230 & -6.95 & vdW: Lys102 (A), Lys103 (A), Tyr181 (A), Phe227 (A), His235 (A) & Lys101 (A), lle180 (A) Glu138 (B), Leu100 (A), Pro236 (A) \\
\hline & & & $\begin{array}{l}\text { Pi interactions: Leu100 (A), VAL106 (A), Tyr188 (A), Val179 (A), Leu } \\
234 \text { (A) }\end{array}$ & 5 \\
\hline \multirow[t]{3}{*}{ MR } & 120 & -5.02 & $\begin{array}{l}\text { vdW: Pro95 (A), Lys102 (A), Thr139 (B), Lys172 (A), Tyr188 (A), } \\
\text { Phe227 (A), Trp229 (A), Leu234 (A), His235 (A) }\end{array}$ & Lys101 (A), Ile108 (A), Tyr318 (A) \\
\hline & & & Pi interactions: Leu100 (A), Tyr181 (A), GLU138 (B), VAL179 (A) & 4 \\
\hline & & & Alkil interactions: Lys102 (A), Val106 (A), Pro236 (A) & \\
\hline \multirow[t]{2}{*}{ MRG } & 45 & 2.38 & $\begin{array}{l}\text { vdW: Glu } 28 \text { (B) Lys } 102 \text { (A), Lys } 103 \text { (A), Val } 106 \text { (A), le } 135 \text { (A), } \\
\text { Val } 179 \text { (A), Tyr } 181 \text { (A), Tyr } 188 \text { (A), Gly } 190 \text { (A) Asp } 192 \text { (A), Asp } \\
320 \text { (A) }\end{array}$ & Lys101, Glu138 \\
\hline & & & Pi interactions: Leu 100 (A), Pro 321 (A) & 2 \\
\hline
\end{tabular}

$M$ myricetin, MR myricetin rhamnoside, MRG myricetin 3-(6-rhamnosylgalactoside). EC50: Antiviral activity ( $\mu \mathrm{M})$ measured by Elisa p24, EB free energy of binding (kcal/ $\mathrm{mol}), v d W$ van der Waals interactions

a Note that the more positive the free energy of binding, the less likely the probability interaction 


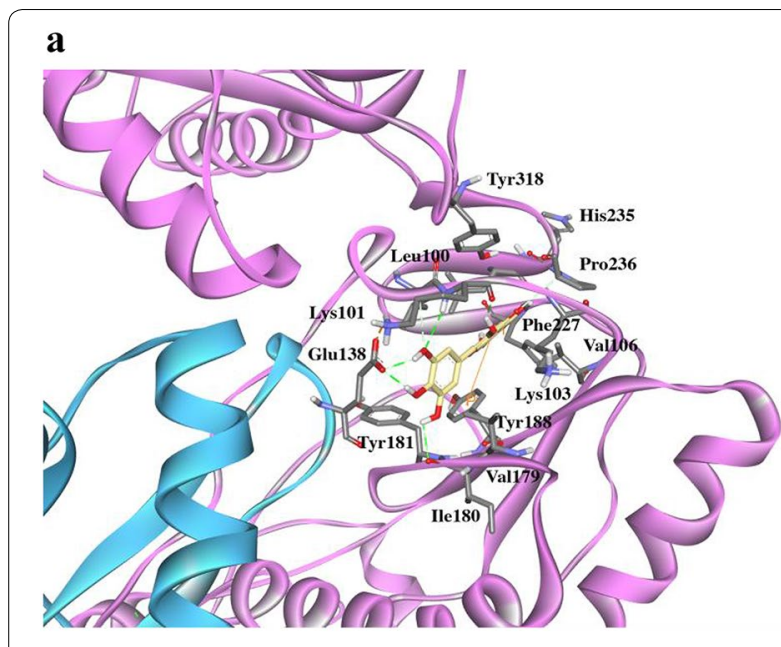

b

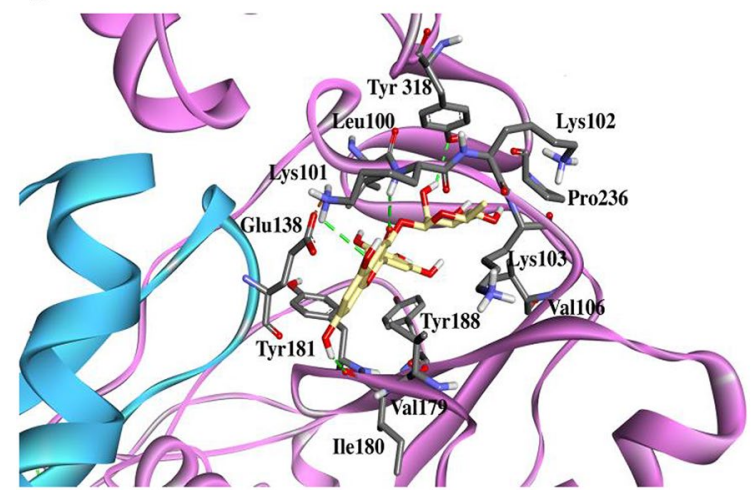

Fig. 4 Docking results. Best pose of $M \mathbf{a}$ and $M R \mathbf{b}$ into the no nucleoside binding pocket of HIV-1 RT. The ligand is shown in gold and residues involved in the interactions in gray. The hydrogen bonds are represented as a green dotted line

Trp229, Leu234, and Tyr318 of the p66 sub-unit and Glu138 of the p51 sub-unit. The analysis of the docked complexes emphasizes the relevant role of the $\mathrm{H}$-bonds formed with residues Lys101, Ile 108, Ile 180, Tyr 318 and Glu 318. The results presented in this work are in agreement with those obtained by Syahdi in 2012 [31], which evaluated HIV RT docking of other flavonoids derived from plants and found that a hydrogen bond interaction with Lys101 is present on the docked pose with the lowest free energy of binding. From the docking analysis, myricetin might interact through five hydrogen bonds, while myricetin 3-rhamnoside through four hydrogen bonds and myricetin 3-(6-rhamnosylgalactoside) through only two. The number of hydrogen bonds formed is related to the free energy of binding found for each compound, and those molecules that can form a greater number of hydrogen bonds consequently create a more stable interaction.
Myricetin and the derivatives evaluates in this study have not been tested against HIV-1 resistant strains, nor mutant enzymes, in vitro to evaluate their capacity to inhibit viral replication of NNRTI resistant viruses. However, the docking study of myricetin on a RT enzyme with mutations conferring NNRTI resistance (L101I, K103N, and E138K) was performed with the protein models $1 \mathrm{~s} 1 \mathrm{u}$, $1 \mathrm{fkp}$ and $2 \mathrm{hny}$ respectively. The analysis showed that the mutations did not result in significant changes in the free energy of binding levels, although with the L101I mutation, a small change in the orientation of myricetin in the binding pocket was observed. To better understand the possible effect of mutations, our laboratory is currently studying the interaction of myricetin in the NNRTI binding pocket through molecular dynamics simulations. We will also perform an in vitro analysis with NNRTI resistant strains of HIV-1 to determine the effect of the mutations on inhibitory action of these flavonoids derivatives.

\section{Conclusions}

The flavonoids are produced naturally as glycosides in plants and fruits but are found as aglycones in blood, as a consequence of the hydrolysis by glycosidases at the intestine lumen or inside the cell. The glycosylated flavonoids could be actively transported by SGLT1, with posterior hydrolysis within cytoplasm or they could be hydrolyzed at the cell surface and then enter the cell by diffusion. The existence of two different chemical forms of the flavonoid could generate a gradient constant favoring an increased concentration inside the cell.

The differences in the antiviral activity and the RT inhibition of the myricetin, MR and MRG together with the in silico analysis suggest that the glycosyl moiety could play a role in the entry of flavonoids into the cell and then, after enzymatic cleavage of the glycosyl moiety, the myricetin aglycone would ultimately be responsible for anti-HIV activity. This activity is presumed to be mediated primarily by the inhibition of RT, although interaction with other targets can't be discarded with the results described. Further studies are required to clarify the role of the glycosides in the activity/transport of myricetin and other related flavonoids.

\section{Abbreviations}

HIV: human immunodeficiency virus; RT: reverse transcriptase; MOI: multiplicity of infection; M: myricetin; MR: myricetin 3-rhamnoside; MRG: myricetin 3-(6-rhamnosylgalactoside); EC50: half effective concentration; CC50: half cytotoxic concentration; IC50: half inhibitory concentration.

\section{Authors' contributions}

This study was conceived and designed by JTO, HRR, MLS and FHP. JTO, HRR and MLS implemented the study and analyzed results. AIS and JB purified and characterized the compounds. JTO and HRR drafted the manuscript. All authors read and approved the final manuscript. 


\begin{abstract}
Author details
${ }^{1}$ Laboratorio de Virología Molecular, Centro de Microbiología y Biología

Celular, Instituto Venezolano de Investigaciones Científicas, Caracas, Venezuela.

${ }^{2}$ Laboratorio de Productos Naturales, Facultad de Farmacia, Universidad Central de Venezuela, Caracas, Venezuela. ${ }^{3}$ Unidad de Química Medicinal, Facultad de Farmacia, Universidad Central de Venezuela, Caracas, Venezuela.
\end{abstract}

\section{Acknowledgements}

We thanks to Dr. José Domingo Medina for providing Myricetin. Also, we thanks to Dr. Stephen Tillett from Herbario Víctor Manuel Ovalles, Pharmacy School (Universidad Central de Venezuela) for the botanical identification and Dr Howard Takiff for the revision of English.

\section{Competing interests}

The authors declare that they have no competing interests.

\section{Availability of data and materials}

All data generated or analyzed during this study are included in this published article.

\section{Consent for publication}

Not applicable.

\section{Ethics approval and consent to participate}

Not applicable.

\section{Funding}

This study was supported by Grant 495 to HRR, from the Instituto Venezolano de Investigaciones Científicas, Caracas, Venezuela.

\section{Publisher's Note}

Springer Nature remains neutral with regard to jurisdictional claims in published maps and institutional affiliations.

Received: 15 June 2017 Accepted: 4 October 2017

Published online: 12 October 2017

\section{References}

1. UNAIDS. UNAIDS report on the global AIDS epidemic Geneva: Joint United Nations Programme on HIV/AIDS (UNAIDS). 2012. http://wwW. unaids.org/sites/default/files/media_asset/20121120_UNAIDS_Global_ Report_2012 with_annexes en_1.pdf. Accessed Jan 2017.

2. Collaboration Antiretroviral Therapy Cohort. Life expectancy of individuals on combination antiretroviral therapy in high-income countries: a collaborative analysis of 14 cohort studies. Lancet. 2008;372(9635):293-9.

3. Cihlar T, Fordyce M. Current status and prospects of HIV treatment. Curr Opin Virol. 2016;26(18):50-6.

4. Mukhtar M, Arshad M, Ahmad M, Pomerantz RJ, Wigdahl B, Parveen Z. Antiviral potentials of medicinal plants. Virus Res. 2008;131:111-20.

5. Naithani R, Huma LC, Holland LE, Shukla D, McCormick DL, Mehta RG, Moriarty RM. Antiviral activity of phytochemicals: a comprehensive review. Mini Rev Med Chem. 2008:8(11):1106-33.

6. Orhan DD, Ozçelik B, Ozgen S, Ergun F. Antibacterial, antifungal, and antiviral activities of some flavonoids. Microbiol Res. 2010;165(6):496-504.

7. Rathee P, Chaudhary H, Rathee S, Rathee D, Kumar V, Kohli K. Mechanism of action of flavonoids as anti-inflammatory agents: a review. Inflamm Allergy Drug Targets. 2009;8(3):229-35.

8. Hokche DO, Ramírez N. Sistemas reproductivos en especies de Melastomataceae en la Gran sabana (Estado Bolívar, Venezuela). Acta Botanica Venezuelica. 2008:31:387-408.

9. Berry PE, Luckana N. Melastomataceae. In: Berry PE, Yatskievych K, Holst BK, editors. Flora of the Venezuelan Guayana. Liliaceae-Myrsinaceae. 6th ed. Louis: Missouri Botanical Garden, St; 2001. p. 263-383.
10. Leite TC, de Sena AR, Dos Santos Silva TR, Dos Santos AK, Uetanabaro AP, Branco A. Antimicrobial activity of Marcetia DC species (Melastomataceae) and analysis of its flavonoids by reverse phase-high performance liquid chromatography coupled-diode array detector. Pharmacogn Mag. 2012:8(31):209-14.

11. Pasetto S, Pardi V, Murata RM. Anti-HIV-1 activity of flavonoid myricetin on HIV-1 infection in a dual-chamber in vitro model. PLoS ONE. 2014;29:e115323

12. Ono K, Nakane H, Fukushima M, Chermann JC, Barré-Sinoussi F. Differential inhibitory effects of various flavonoids on the activities of reverse transcriptase and cellular DNA and RNA polymerases. Eur J Biochem. 1990;190(3):469-76

13. De Clercq E. Antiretroviral drugs. Curr Opin Pharmacol. 2010;10(5):507-15.

14. Li S, Hattori T, Kodama EN. Epigallocatechin gallate inhibits the HIV reverse transcription step. Antivir Chem Chemother. 2011;21(6):239-43.

15. Sluis-Cremer N, Tachedjian G. Mechanisms of inhibition of HIV replication by nonnucleoside reverse transcriptase inhibitors. Virus Res. 2008:134(1-2):147-56.

16. Ortega JT, Estrada O, Serrano M, Contreras W, Orsini G, Pujol FH, Rangel HR. Glycosylated flavonoids from Psidium guineense as major inhibitors of HIV replication in vitro. Nat Prod Commun. 2017;12(7):1049-52.

17. Baptista J, Chavez K, Torrico F, Trejo E, García C, Urbina J, Carrasco J, Tadde A, Tillet S, Suarez Al. Constituyentes químicos y actividad antiinflamatoria de Marcetia taxifolia. CIENCIA. 2016:24(2):82-94.

18. Quintero A, Fabbro R, Maillo M, Barrios M, Milano MB, Fernández A, Williams B, Michelangeli F, Rangel HR, Pujol FH. Inhibition of hepatitis $B$ virus and human immunodeficiency virus (HIV-1) replication by Warscewiczia coccinea (Vahl) Kl. (Rubiaceae) ethanol extract. Nat Prod Res. 2011:25(16):1565-9.

19. Berman H, Westbrook J, Feng G, Gilliland T, Bhat H, Weissig IN, Shindyalov PE. Bourne: the protein data bank. Nucleic Acids Res. 2000:28:235-42.

20. Das K, Martinez SE, Bauman JD, Arnold E. HIV-1 reverse transcriptase complex with DNA and nevirapine reveals non-nucleoside inhibition mechanism. Nat Struct Mol Biol. 2012;19(2):253-9.

21. Phillips J, Braun R, Wang W, Gumbart J, Tajkhorshid E, Villa E, Chipot C, Skeel R, Kale L, Schulten K. Scalable molecular dynamics with NAMD. J Comput Chem. 2005;26:1781-802

22. Kim S, Thiessen PA, Bolton EE, Chen J, Fu G, Gindulyte A, Han L, He J, He S, Shoemaker BA, Wang J, Yu B, Zhang J, Bryant SH. PubChem substance and compound databases. Nucleic Acids Res. 2016:44(D1):D1202-13.

23. Jeong JJ, Kim DH. 5,7-Dihydroxy-6-methoxy-flavonoids eliminate HIV-1 D3-transfected cytoprotective macrophages by inhibiting the PI3K/Akt signaling pathway. Phytother Res. 2015. doi:10.1002/ptr.5388.

24. Namazi R, Zabihollahi R, Behbahani M, Rezaei A. Inhibitory activity of avicennia marina, a medicinal plant in persian folk medicine, against HIV and HSV. Iran J Pharm Res. 2013;12(2):435-43.

25. Sauter D, Schwarz S, Wang K, Zhang R, Sun B, Schwarz W. Genistein as antiviral drug against HIV ion channel. Planta Med. 2014;80(8-9):682-7.

26. Serra H, Mendes T, Bronze MR, Simplicio AL. Prediction of intestinal absortion and metabolism of pharmacologically active flavones and flavanones. Bioorg Med Chem. 2008;16:4009-18.

27. Walgren RA, Lin JT, Kinne RK, Walle T. Cellular uptake of dietary flavonoid quercetin 4'-beta-glucoside by sodium-dependent glucose transporter SGLT1. J Pharmacol Exp Ther. 2000;294(3):837-43.

28. Passamonti S, Terdoslavich M, Franca R, Vanzo A, Tramer F, Braidot E, Petrussa E, Vianello A. Bioavailability of flavonoids: a review of their membrane transport and the function of bilitranslocase in animal and plant organisms. Curr Drug Metab. 2009;10:369-94.

29. Tapiero H, Tew KD, Naguyen Ba G, Mathé G. Polyphenols: do the play a role in the prevention of human pathologies? Biomed Phamacother. 2002;56:200-2007.

30. Sarafianos SG, Marchand B, Das K, Himmel DM, Parniak MA, Hughes $\mathrm{SH}$, Arnold E. Structure and function of HIV-1 reverse transcriptase: molecular mechanisms of polymerization and inhibition. J Mol Biol. 2009;385(3):693-713.

31. Syahdi RR, Mun'im A, Suhartanto H, Yanuar A. Virtual screening of Indonesian herbal database as HIV-1 reverse transcriptase inhibitor. Bioinformation. 2012:8(24):1206-10. 